\title{
Postcoordination of LOINC Codes in SNOMED CT
}

\author{
Nina HAFFER ${ }^{\mathrm{a}, \mathrm{b}, 1}$ and Sylvia THUN ${ }^{\mathrm{b}, \mathrm{c}, \mathrm{d}}$ \\ ${ }^{a}$ Hochschule für Technik und Wirtschaft (HTW) - University of Applied Sciences, \\ Berlin, Germany \\ ${ }^{\mathrm{b}}$ Berlin Institute of Health (BIH), Germany \\ ${ }^{\mathrm{c}}$ Charité - Universitätsmedizin Berlin, Germany \\ ${ }^{\mathrm{d}}$ Hochschule Niederrhein - University of Applied Sciences, Krefeld, Germany
}

\begin{abstract}
The objectives of this paper are to analyze the terminologies SNOMED CT and Logical Observation Identifiers Names and Codes (LOINC) and to provide a guideline for the translation of LOINC concepts to SNOMED CT. Verified research data sets were used for this study, so this experiment is replicable with other research data. 50 LOINC concepts of frequently performed laboratory services were translated to SNOMED CT. Information would be lost with pre-coordinated mapping but the compositional grammar of SNOMED CT allows for the linking of individual concepts into complicated postcoordinated expressions including all embedded information in LOINC concepts. All information can thus be transferred smoothly to SNOMED CT.
\end{abstract}

Keywords. Semantic interoperability, SNOMED CT, LOINC, Mapping

\section{Introduction}

Terminology standards are considered an indispensable requirement for semantic interoperability. They provide universal, uniquely assignable identifiers that enable the exchange of clinical data between heterogeneous computer systems.

In German laboratories, clinical information is marked with in-house and manufacturer-specific identification codes and therefore cannot be recognized by other systems with their own designations and abbreviations. In order to make laboratory data semantically interoperable, a common understanding of information units exchanged between the systems is needed. The semantic standardization of information regarding laboratory analyses - the commissions and results - is achieved by data migration of inhouse encodings to internationally recognized, terminologies [1]. In this way, data is not only technically processed. It is also assigned a clear meaning across systems.

Logical Observation Identifier Names and Codes (LOINC) and Systematized Nomenclature of Medicine - Clinical Terms (SNOMED CT) are suitable for the standardization of laboratory analyses. LOINC is a simple database developed especially for coding laboratory data. SNOMED CT is the most complex terminology standard in the medical field due to its ontological properties and compositional grammar.

\footnotetext{
${ }^{1}$ Corresponding Author, Nina Haffer, Berlin Institute of Health (BIH), Anna-Louisa-Karsch-Strasse 2, 10178 Berlin, Germany; E-mail: nina.haffer@web.de.
} 
Because of the wide range of usages of SNOMED CT, it is possible to translate LOINC codes into SNOMED CT using proprietary guidelines. However, there are few recent studies that analyze whether LOINC concepts can be translated to SNOMED CT without loss of information (but see $[2,3,4]$ ). The aim of this study is to translate LOINC concepts to SNOMED CT to demonstrate the precision with which LOINC concepts can be represented via the ontology. With SNOMED CT, hierarchical structures can be added and encode information with greater precision.

\section{Methods}

The content and structure of the SNOMED CT and LOINC terminologies are specified in the manufacturer's own guidelines [1,5]. The July 2019 international edition of SNOMED CT and LOINC version 2.66 were used.

\subsection{LOINC coded research data}

For this study, a validated LOINC encoded research data set was utilized. The data was sourced from the 50 most performed services of 2017 from a local laboratory. Laboratories code their services for clinics and practices with their own performance identifiers. Each performance ID is specific to each analysis. For this reason, no concepts encoding laboratory orders were used for LOINC coding, only codes that are intended for the presentation of results. The data set included both single and panel codes. Before migration to SNOMED CT, the LOINC annotations were quality assured according to the requirements for mappings in ISO/TS 21564:2019 [6].

\subsection{Arising information gaps in pre-coordinated SNOMED CT concepts}

Via the domains procedures and observable entities, activities in the field of healthcare can be coded in SNOMED CT, including diagnostic procedures [7]. Although the terminology is very broad and covers many parts of laboratory diagnostics, LOINC codes cannot be replaced by pre-coordinated mapping with SNOMED CT because not all information of the multi-axial LOINC concepts is included (Table 1).

Table 1. Pre-coordination of a LOINC concept with SNOMED CT: Not all information is included, such as the measured parameter, the method (1) or a wrong type of sample is listed (2-5).

\begin{tabular}{|l|l|l|r|}
\hline \multicolumn{1}{|c|}{ LOINC } & \multicolumn{1}{|c|}{ SNOMED CT } & \multicolumn{1}{|c|}{ ID } \\
\hline \multirow{3}{*}{$\begin{array}{l}\text { Leukocytes } \\
\text { in } \begin{array}{l}\text { Z } \\
\text { Automated } \\
\text { count }\end{array}\end{array}$} & White blood cell count (procedure) & $6690-2$ & \\
\cline { 2 - 4 } & $\begin{array}{l}\text { White blood cell count, automated, cerebrospinal fluid } \\
\text { (procedure) }\end{array}$ & 767002 & $(1)$ \\
\cline { 2 - 4 } & $\begin{array}{l}\text { White blood cell count, automated, peritoneal fluid } \\
\text { (procedure) }\end{array}$ & 104115009 & $(3)$ \\
\cline { 2 - 4 } & White blood cell count, automated, pleural fluid (procedure) & 104119003 & $(4)$ \\
\cline { 2 - 4 } & White blood cell count, automated, semen (procedure) & 104124000 & $(5)$ \\
\hline
\end{tabular}

\subsection{Postcoordination of LOINC concepts in SNOMED CT}

Using logic operators and appropriate attributes, the LOINC axes were therefore manually mapped, using postcoordination to SNOMED CT. The mapping was validated 
by two map specialists according to the requirements for mappings in ISO/TS 21564:2019 [6].

Attributes suggested by SNOMED CT were used for the attribute relationships [5]. For the documentation of laboratory analyses, concepts from the domain Observable Entity for focus concepts were used. However, if no suitable concepts from the level were available, the concept of the same name |Observable Entity| was used.

Many concepts describing procedures belong to the domain Procedure, which is based on the precise ontological differentiation of the domains in SNOMED CT [6]. The domain Procedure refers to the technical execution of a laboratory test or other examination. Observable entities abstract laboratory analyses as 'observations', which can also have a result. The attributes define the focus concept in more detail (Table 2).

Table 2. Attributes defining postcoordinated concepts and explaining their functions.

\begin{tabular}{|l|l|}
\hline Concept & Function \\
\hline $\mid$ Component $\mid$ & Component observed or measured by a laboratory test or procedure \\
\hline $\mid$ Property type $\mid$ & Specification of the type of property to be measured \\
\hline $\mid$ Time aspect $\mid$ & Definition of temporal relations \\
\hline $\mid$ Direct site $\mid$ & $\begin{array}{l}\text { Place or sample at which the observation takes place, i.e. the observation } \\
\text { system }\end{array}$ \\
\hline $\mid$ Inheres in $\mid$ & $\begin{array}{l}\text { In the observation system is an entity in which the property to be } \\
\text { observed is embedded, for example: A serum sample is used to } \\
\text { determine the sodium concentration in body plasma. For plasma, Inheres } \\
\text { in is used, for serum Direct site } \mid\end{array}$ \\
\hline $\mid$ Scale Type $\mid$ & Scaling the result of an observation \\
\hline $\mid$ Technique $\mid$ & $\begin{array}{l}\text { Description of the method by which a laboratory analysis is carried out } \\
\text { and more detailed description of the test }\end{array}$ \\
\hline $\mid$ Adjustment $\mid$ & $\begin{array}{l}\text { Additional references to analytical techniques in both the component and } \\
\text { method axes }\end{array}$ \\
\hline
\end{tabular}

For the system axis, sometimes more than one attribute relationship is used. Since clinical chemistry often uses LOINC terms such as Ser/Plas (1) or PPP (2), special rules are provided for mapping in SNOMED CT (Table 3).

Table 3. The LOINC system axis in SNOMED CT.

\begin{tabular}{|c|c|}
\hline $\begin{array}{l}(704327008 \mid \text { Direct site } \mid= \\
\text { 704319004|Inheres in } \mid=\end{array}$ & $\begin{array}{l}\text { 122592007|Acellular blood (serum or plasma) specimen|, } \\
50863008 \text { (Plasma) }\end{array}$ \\
\hline $\begin{array}{l}(704327008 \mid \text { Direct site } \mid= \\
704319004 \mid \text { Inheres in }=\end{array}$ & $\begin{array}{l}\text { 119362004|Platelet poor plasma specimen|, } \\
\text { 50863008(Plasma) }\end{array}$ \\
\hline
\end{tabular}

Postcoordinated SNOMED CT codes are schematically structured as follows (Table 4). Only concepts from the displayed top-level hierarchies are used for postcoordination. In the specification of SNOMED CT the attribute |Technique|, which is also used for the representation of the method axis, has been suggested for adjustments [8]. But since this leads to erroneous semantics when methods are already listed under |Technique| in laboratory analyses, the attribute |Adjustment| was used for postcoordination ${ }^{2}$.

\footnotetext{
${ }^{2}$ See Results (3), (5)
} 
Table 4. Required hierarchical affiliations for attribute-value relationships.

\begin{tabular}{|c|c|c|}
\hline \multicolumn{2}{|c|}{ Focus concept } & Observable Entity \\
\hline \multirow[t]{7}{*}{ Attribute } & Component (attribute) $=$ & $\begin{array}{l}\text { Body structure| OR } \\
\text { Organism| OR } \\
\text { |Substance OR } \\
\text { Specimen| OR } \\
\text { Physical object| OR } \\
\text { Pharmaceutical/biologic product| OR } \\
\text { Record artifact }\end{array}$ \\
\hline & Adjustment (attribute) $=$ & $\begin{array}{l}\text { Qualifier value| OR } \\
\text { Procedure }\end{array}$ \\
\hline & Property type (attribute) $=$ & Qualifier value \\
\hline & Direct site $($ attribute $)=$ & Specimen \\
\hline & Inheres in (attribute) $=$ & Substance \\
\hline & Scale type (attribute) $=$ & Qualifier value \\
\hline & Technique (attribute) $=$ & Procedure \\
\hline
\end{tabular}

\section{Results}

Five typical examples of the 50 postcoordinated terms are listed below with associated LOINC terms to illustrate the logical structure of a postcoordinated term (Table 5). These examples serve the translational reasoning, so that the knowledge gained from the examples can be applied to all other postcoordinated terms.

\section{Discussion}

The approach of LOINC is simple. No ontological dependencies are created in addition to sub-specializations and documentation standards. The terminology also covers different document types. Over $90 \%$ of the local Health Information Systems documents can be annotated with a LOINC code indicating that the terminology is suitable for the standardization of local health documents [9]. Activities to expand the LOINC database with hierarchical structures have only recently begun, so that the applicability is limited to the identification of laboratory analyses using language-independent codes [10]. However, there is a demand to enrich LOINC with hierarchical structures in order to extend the terminology to an ontology and thus increase the semantic interoperability of the terminology [11]. SNOMED CT, on the other hand, is a more diversified terminology with ontological dependencies. Compared to LOINC, the precision is higher. It follows a complex logical model, according to which SNOMED CT components are connected to each other. 
Table 5. Juxtaposing LOINC concepts with postcoordinated SNOMED CT terms.

\begin{tabular}{|c|c|c|c|c|c|c|}
\hline \multirow{2}{*}{$\frac{\text { LOINC }}{58410-2}$} & Component & Property & Time & System & Scale & Method \\
\hline & $\begin{array}{l}\text { Complete blood count } \\
\text { (hemogram) panel }\end{array}$ & - & Pt & Bld & - & $\begin{array}{l}\text { Automated } \\
\text { count }\end{array}$ \\
\hline \multicolumn{7}{|c|}{ SNOMED CT } \\
\hline \multicolumn{7}{|c|}{ 363787002|Observable entity|: } \\
\hline \multicolumn{7}{|c|}{$\begin{array}{l}\text { (246093002|Component }|=63370004| \text { Blood cell } \mid, \\
370134009 \mid \text { Time aspect }|=123029007| \text { Single point in time } \mid, \\
704327008 \mid \text { Direct site }|=258580003| \text { Whole blood sample } \mid, \\
246501002 \mid \text { Technique }|=117356000| \text { Blood cell count, automated } \mid)\end{array}$} \\
\hline LOINC & Component & Property & Time & System & Scale & Method \\
\hline $6690-2$ & Leukocytes & NCnc & $\mathrm{Pt}$ & Bld & Qn & $\begin{array}{l}\text { Automated } \\
\text { count }\end{array}$ \\
\hline \multicolumn{7}{|c|}{ SNOMED CT } \\
\hline \multicolumn{7}{|c|}{ 363787002|Observable entity|: } \\
\hline & \multicolumn{6}{|c|}{$\begin{array}{l}(246093002 \mid \text { Component }|=52501007| \text { Leukocyte } \mid \\
246093002 \mid \text { Property }|=118550005| \text { Number concentration (property) } \mid, \\
370134009 \mid \text { Time aspect }|=123029007| \text { Single point in time } \mid, \\
704327008 \mid \text { Direct site }|=258580003| \text { Whole blood sample } \mid, \\
370132008 \mid \text { Scale type }|=30766002| \text { Quantitative } \mid, \\
246501002 \mid \text { Technique }|=| 121101003 \mid \text { Automated cell count method } \mid)\end{array}$} \\
\hline \multirow{2}{*}{$\frac{\text { LOINC }}{49701-6}$} & Component & Property & Time & System & Scale & Method \\
\hline & $\begin{array}{l}\mathrm{pH}^{\wedge \wedge} \text { adjusted to patient's } \\
\text { actual temperature }\end{array}$ & LsCnc & $\mathrm{Pt}$ & Bld & Qn & - \\
\hline \multicolumn{7}{|c|}{ SNOMED CT } \\
\hline \multicolumn{7}{|c|}{ 363787002|Observable entity|: } \\
\hline & \multicolumn{6}{|c|}{$\begin{array}{l}\text { (246093002 } \mid \text { Component }|=89177007| \text { Proton } \mid \\
410616005 \mid \text { Adjustment }|=707688001| \text { Adjusted to patients actual temperature technique } \mid \text {, } \\
246093002 \mid \text { Property }|=702700007| \text { Logarithmic substance concentration (property) } \mid \\
370134009 \mid \text { Time aspect }|=123029007| \text { Single point in time } \mid \\
704327008 \mid \text { Direct site }|=258580003| \text { Whole blood sample } \mid \\
370132008 \mid \text { Scale type }|=30766002| \text { Quantitative } \mid \\
246501002 \mid \text { Technique }|=81065003| \text { pH measurement } \mid \text { ) }\end{array}$} \\
\hline LOINC & Component & Property & Time & System & Scale & Method \\
\hline $14118-4$ & Lactate & MCnc & $\mathrm{Pt}$ & Ser/Plas & Qn & - \\
\hline \multicolumn{7}{|c|}{ SNOMED CT } \\
\hline \multicolumn{7}{|c|}{ 363787002|Observable entity|: } \\
\hline & \multicolumn{6}{|c|}{$\begin{array}{l}\text { (246093002|Component }|=83036002| \text { Lactate } \mid \text {, } \\
246093002 \mid \text { Property }|=118539009| \text { Mass concentration (property) } \mid, \\
370134009 \mid \text { Time aspect }|=123029007| \text { Single point in time } \mid, \\
704327008 \mid \text { Direct site }|=122592007| \text { Acellular blood (serum or plasma) specimen } \mid \text {, } \\
704319004 \mid \text { Inheres in }|=50863008| \text { Plasma } \mid \\
370132008 \mid \text { Scale type }|=30766002| \text { Quantitative } \mid \\
246501002 \mid \text { Technique }|=3926003| \text { Lactic acid measurement } \mid)\end{array}$} \\
\hline LOINC & Component & Property & Time & System & Scale & Method \\
\hline $6301-6$ & $\begin{array}{l}\text { Coagulation tissue factor } \\
\text { induced.INR }\end{array}$ & RelTime & $\mathrm{Pt}$ & PPP & Qn & Coag \\
\hline \multicolumn{7}{|c|}{ SNOMED CT } \\
\hline \multicolumn{7}{|c|}{ 103220009|Tissue factor induced coagulation|: } \\
\hline & \multicolumn{6}{|c|}{$\begin{array}{l}\text { (246093002 } \mid \text { Component }|=387124011| \text { Thromboplastin } \mid, \\
410616005 \mid \text { Adjustment }|=707688001| \text { Calculation of international normalized ratio|, } \\
246093002 \mid \text { Property }|=118539009| \text { Relative time (property) } \mid \\
370134009 \mid \text { Time aspect }|=123029007| \text { Single point in time } \mid \\
704327008 \mid \text { Direct site }|=119362004| \text { Platelet poor plasma specimen } \mid, \\
704319004 \mid \text { Inheres in }|=50863008| \text { Plasma } \mid \\
370132008 \mid \text { Scale type }|=30766002| \text { Quantitative } \mid \\
246501002 \mid \text { Technique }|=119266006| \text { Coagulation } \mid \text { ) }\end{array}$} \\
\hline
\end{tabular}


When comparing the two terminologies, it becomes clear that SNOMED CT has at least as much potential as LOINC regarding the standardization of laboratory data. Additionally, codes from Unified Codes for Units of Measure (UCUM) could be used to define the unit of each measurement [12]. The six axes that make up LOINC codes were migrated to SNOMED CT without any loss of information. Thus, SNOMED CT covers the conceptual information and compositional semantics of LOINC, so that postcoordinated SNOMED CT expressions can be considered semantically equivalent to LOINC codes. Though, to represent the sub-specifications of LOINC in SNOMED CT, the data needed to encode the information could e.g. be further adjusted using Reference Sets in SNOMED CT to expand the concept relations of SNOMED CT [13]. It can be assumed that a large proportion of laboratory procedures can be covered by few codes [14]. But note that the number of postcoordinated terms should be extended in the future in order to derive specific guidelines. In addition, manual coding is relatively timeconsuming and it would be desirable to have automated processes for translating LOINC codes in SNOMED CT in the future [10].

There is also a guideline in the form of a cooperation agreement that describes how to combine SNOMED CT and LOINC by efficient work sharing. The cooperation makes standard use of the joint specifications of SNOMED International and Regenstrief Institute [15].

It also appears that the SNOMED CT guidelines for postcoordination are not sufficiently maintained, which makes a generally valid procedure for the mapping of LOINC concepts in SNOMED CT difficult. In the specification of SNOMED CT it has been proposed to use |Property Type| and not |Property| as an attribute. However, |Property Type| is an outdated concept and therefore no longer part of the implementable SNOMED CT codes [16].

SNOMED International advocates the use of LOINC codes to represent requirements and observations in countries where LOINC has been adopted as a standard [17]. The agreement also describes the most common pattern for collaboration: LOINC provides codes that represent the names of information items (e.g., questions), and SNOMED CT provides codes that encode nominal and order values (e.g., answers) [15].

As a result of this cooperation, The LOINC/SNOMED CT Expression Association and Map Sets Files were created [18]. However, these are based on the July 2017 international edition of SNOMED CT and LOINC version 2.58 and are therefore not up to date.

In example (5) in the results, International Normalized Ratio, INR for short, is given as an adjustment of the examination parameters. This is determined by means of the socalled thromboplastin time, which in turn is specified in the measured variable-coding attribute 'property' and not in the method axis. The coagulation process of the blood is specified in this axis. Combining the codes in one attribute would merge different semantic meanings which do not belong together in one axis. If, on the other hand, no entries are made in LOINC on certain axes at all, the corresponding attributes are also omitted in SNOMED CT ${ }^{3}$.

For the postcoordination in SNOMED CT a concept from the domain Observable Entity is used as the focus concept. That can be a concept that allows a more detailed description of the procedure or the generally valid concept |Observable entity|, which is at the top of the hierarchical level. SNOMED CT codes, which would define the LOINC concept more precisely in the focus concept, are usually found in the procedure domain

\footnotetext{
${ }^{3}$ See Results (1)
} 
|Procedure|. An example of this is the automated leukocyte counting. If |White blood cell count| were selected from the procedure domain as the superordinate focus concept, the component axis would be omitted, since it would already be present in the superordinate concept. Furthermore, the attribute relationship between the attribute |Component| and the concept |Leukocyte| to the focus concept |White blood cell count| would be in an ontological dependency.

In addition to the simplifications of the postcoordinated term if the scaling axis was eliminated ${ }^{4}$, the attribute relationship for the component description would then also be removed. However, this simplification is not allowed by clear differentiations in the semantic and ontological meaning of observable entities and procedures, which is why a data shortening problem arises. So, when considering data reduction in digitization, SNOMED CT requires more concepts to describe an analysis than LOINC, although these are more precise and, if desired, more detailed.

\section{Conclusion}

In postcoordinated terms, all terms can be recorded individually and thus all information can be mapped, which allows for profound coding in SNOMED CT. Compared to LOINC, the precision of statements made is higher. The coding of LOINC concepts in SNOMED CT is specified in SNOMED International guidelines. However, these guidelines need to be updated to be used by operators.

\section{References}

[1] SNOMED International, SNOMED CT Starter Guide, https://confluence.ihtsdotools.org/display/DOCSTART/SNOMED+CT+Starter+Guide [cited 2020 March 25].

[2] S. Santamaria, A. Farzaneh, and K. Spackman, Linking LOINC and SNOMED CT. AMIA Annual Symposium Proceedings 109 (2012). 99-101.

[3] O. Bodenreider. "Issues in mapping LOINC laboratory tests to SNOMED CT." AMIA Annual Symposium Proceedings (2008). 51-55.

[4] P. Ciancarini, F. Poggi, M. Horridge, J. Zhao, T. Groza, M.C. Suarez-Figueroa, M. d'Aquin, and V. Presutti, Knowledge Engineering and Knowledge Management, Springer International Publishing, Cham, 2017. 145-148.

[5] Regenstrief Institute Inc., LOINC Users' Guide, https://loinc.org/download/loinc-users-guide/ [cited 2020 March 25].

[6] ISO/TS 21564:2019: Health Informatics - Terminology resource map quality measures (MapQual), https://www.iso.org/standard/71088.html.

[7] https://confluence.ihtsdotools.org/display/DOCEG/Observable+Entity [cited 2020 March 25].

[8] SNOMED International, SNOMED CT July 2017 LOINC - SNOMED CT Cooperative package Production release - RF2 Release notes, https://oinc.org/file-access/download-id/9516/ [cited 2020 March 25].

[9] M. Dugas, S. Thun, T. Frankewitsch, and K.U. Heitmann, LOINC codes for hospital information systems documents: a case study. J Am Med Inform Assoc 16 (2009), 400-403.

[10] C. Drenkhahn, P. Duhm-Harbeck, and J. Ingenerf, Aggregation and Visualization of Laboratory Data by Using Ontological Tools Based on LOINC and SNOMED CT. Stud Health Technol Inform 264 (2019), 108-112.

\footnotetext{
${ }^{4}$ The result type can already be interpreted as quantitative through the attribute used to describe the property axis
} 
[11] M.C. Lin, D.J. Vreeman, C.J. McDonald, and S.M. Huff, Auditing consistency and usefulness of LOINC use among three large institutions - using version spaces for grouping LOINC codes. Journal of Biomedical Informatics 45 (2012), 658-666.

[12] https://unitsofmeasure.org/ [cited 2020 July 17].

[13] https://confluence.ihtsdotools.org/display/DOCGLOSS/SNOMED+CT+reference+set [cited 2020 March 25].

[14] https://lhncbc.nlm.nih.gov/publication/dataset-loinc-top-300-lab-orders [cited 2020 July 17].

[15] SNOMED International, Using LOINC with SNOMED CT, https://confluence.ihtsdotools.org/display/DOCLOINC/Using+LOINC+with+SNOMED+CT [cited 2020 March 25].

[16] https://confluence.ihtsdotools.org/display/DOCGLOSS/SNOMED+CT+reference+set [cited 2020 March 25].

[17] U.S. National Library of Medicine, New Regenstrief and IHTSDO agreement links LOINC and SNOMED CT to make EMRs more effective at improving health care, 2013.

[18] LOINC/SNOMED CT Expression Association and Map Sets File - Production release (RF2 Format) https://loinc.org/file-access/download-id/9516/ [cited 2020 July 17]. 